\title{
AHÉWA LIKÍ?I: EL RELOJ Y LA JORNADA ENTRE LOS TOBA DEL OESTE DE FORMOSA (GUAYCURÚ, ARGENTINA)
}

\author{
CECILIA PAULA GÓMEZ ${ }^{1}$ \\ CONICET - CIHA \\ MARÍA BELÉN CARPIO ${ }^{2}$ \\ CONICET - UNNE
}

\begin{abstract}
RESUMEN: En este trabajo analizamos las distintas estrategias utilizadas por los grupos toba del oeste de Formosa (Argentina) para identificar los momentos en los que puede dividirse una jornada y la estructura morfosintáctica de las construcciones nominales que los codifican. Se han registrado varios métodos para señalar los lapsos del día, los cuales consisten en la utilización del brazo, un gnomon, "sombras", otros objetos celestes (además del sol), animales y teléfonos celulares. Los segmentos de la jornada más relevantes a ser identificados por cada una de estas estrategias de lectura de tiempo son los que marcan la ida y el regreso al monte, y permiten distinguir dos momentos cualitativamente diferentes pero complementarios: el día y la noche. Demostramos, mediante la articulación de un abordaje etnográfico y lingüístico, que es el sol el indicador de los momentos del día que se asocia inmediatamente al reloj mecánico, indagando en la perspectiva en la que se da esta asociación.
\end{abstract}

PALAVRAS CLAVE: Toba del oeste de Formosa; Guaycurú; Gran Chaco; indicadores de lapsos de la jornada; sol; morfosintaxis.

\section{Ahéwa likíri: Clock and daily routine among the Toba from Western Formosa (Guaicuruan, Argentina)}

ABSTRACT: In this paper, we analyze the strategies used to identify the moments of the day by the Toba from Western Formosa (Argentina), and the morphosyntactic structure of the nominal constructions that encode them. The methods to signal the moments of the daily routine recorded involve the use of the arm, a gnomon, "shades", other celestial objects distinct from the sun,

\footnotetext{
${ }^{1}$ Doctora en Ciencias Sociales por Facultad de Ciencias Sociales de la Universidad de Buenos Aires y Licenciada en Antropología Sociocultural por la Facultad de Filosofía y Letras de la Universidad de Buenos Aires. Actualmente, es investigadora del Consejo Nacional de Investigaciones Científicas y Técnicas (CONICET) y del Centro de Investigaciones Históricas y Antropológicas (CIHA). E-mail: gomezcp@gmail.com .

${ }^{2}$ Doctora en Letras por la Universidad Nacional de Córdoba, Magister en Lingüística por la Universidad de Sonora y Licenciada en Antropología por la Universidad Nacional de Rosario. Actualmente, es investigadora del Consejo Nacional de Investigaciones Científicas y Técnicas (CONICET) y docente en la Universidad Nacional del Nordeste (Argentina). E-mail: belenvenado@yahoo.com.ar .
} 
animals, and cell phones. The most relevant segments of the journey to be identified by each of these indicators of moments of the day are departure to and arrival from the forest. They distinguish two qualitative distinct but complementary moments: day and night. Hence, from an ethnographic and linguistic approach, we demonstrate that the sun is the time indicator that is directly associated to the clock, and study the perspective from which this link is made.

KEYWORDS: Toba from Western Formosa; Guaicuruan; Gran Chaco; indicators of moments of the day; sun; morphosyntax.

\section{Introducción}

Entre los Toba del oeste de Formosa3, el sol es uno de los marcadores que indican los distintos lapsos en los que puede dividirse la jornada; y el reloj mecánico es asociado inmediatamente a él. En este trabajo, describimos y analizamos esta relación desde las perspectivas lingüística y etnográfica. Mediante el análisis lingüístico estudiamos las formas de nombrar el reloj y los distintos momentos del día en esta variedad de toba, y a partir de una aproximación etnográfica, analizamos cómo perciben y seccionan los lapsos en los que se divide la jornada. Ambos caminos confluyen en la relación que se plantea con el sol, lo que nos lleva a tomar una perspectiva que contemple también la construcción del cielo, o más exactamente el camino que haría el sol por el cielo.

Uno de los primeros registros encontrados, hasta el momento, de uso de los relojes mecánicos a los que tuvieron acceso grupos toba del Pilcomayo se encuentra en el testimonio de Francisco Zeballos que ha sido publicado en el Periódico La Estrella de Tarija el 22 de julio de 1882 (COMBÈS, 2017, p. 72). En dicho documento se detalla que parte de las pertenencias que correspondían a los integrantes de la fallida expedición por el río Pilcomayo comandada por Jules Crevaux en 1882 se encontraban en poder de los Toba, siendo los relojes y cronómetros usados como collares. Con el transcurrir del tiempo y tal como otros elementos foráneos que fueron incorporados a su cultura material, el reloj fue adoptado e interpretado teniendo como trasfondo sus propios conocimientos y estrategias para referirse a los diversos instantes en los

\footnotetext{
${ }^{3}$ En este trabajo, cada vez que mencionemos toba sin una aclaración adicional, nos referimos a los Toba del oeste formoseño.
} 
que se divide el transcurso de una jornada. Esta amalgama también se observa en el análisis lingüístico de la forma de referirse al reloj en el idioma toba del oeste de Formosa. El préstamo del español reloj convive con las palabras en idioma toba ahéwa likíi “reloj" (lit. "imagen del sol”), nowácahe "reloj de alguien" (lit. "entidad femenina relacionada con la mano") e yikatená "mi reloj", "revista", "computadora" (lit. "mi objeto femenino que miro"). Actualmente, el reloj mecánico o digital, junto con el celular, dan una pauta acerca de los momentos en los que puede dividirse el día.

El trabajo se organiza de la siguiente manera: en la sección 2 presentamos una descripción de los Toba del oeste formoseño y de aspectos generales de su lengua y en la sección 3 se incluyen antecedentes sobre el recurso al sol como indicador de los momentos del día en pueblos indígenas de Sudamérica. En la sección 4 analizamos la morfosintaxis de los nombres que se refieren al reloj en la variedad de toba estudiada, con especial atención a la construcción idiomática ahéwa likíii "reloj" (lit. "imagen del sol”). Describimos, en la sección 5, las estrategias para leer los distintos momentos del día entre los Toba y por último, en la sección 6 , analizamos los nombres, actividades y significados de los lapsos en los que dividen la jornada. Por último, presentamos reflexiones finales y perspectivas de investigación a futuro.

\section{Los Toba del oeste formoseño y su lengua}

A fines del siglo XIX y principios del XX, comienza la conquista militar del Chaco. La campaña militar que llegó hasta los territorios cercanos al río Pilcomayo, donde solían moverse los grupos toba a los que nos referimos en este trabajo, fue dirigida por el comandante Rostagno en 1911. En la literatura etnográfica estos indígenas han sido denominados Toba-Pilagá, Toba de Sombrero Negro, Toba Ñachilamole?k o Toba del Pilcomayo Medio. Su autodenominación es Qomle?k. Actualmente, estos grupos toba se asientan en poblaciones rurales a sesenta kilómetros de la ciudad de Ingeniero N. Juárez (Departamento ${ }^{4}$

\footnotetext{
${ }^{4}$ Los departamentos constituyen una división territorial de segundo orden dentro de cada provincia que integra el país, excepto en la provincia de Buenos Aires y en la Ciudad Autónoma de Buenos Aires, donde
} 
Matacos, Formosa, Argentina) y también en un barrio que está ubicado en los alrededores de la antedicha ciudad.

A las relaciones interétnicas que tenían estos grupos con sus vecinos Wichís y los más lejanos Pilagás hay que agregarle el contacto con los criollos, los misioneros anglicanos de la South American Missionary Society, que tuvieron una misión entre ellos desde 1930 hasta 1982, y la relación laboral trabada en los ingenios azucareros, a los que migraron como mano de obra estacional aproximadamente desde comienzos del siglo XX. Estuvieron en el ingenio Ledesma, La Esperanza y San Martín del Tabacal; este movimiento migratorio hacia los ingenios se interrumpió a fines de la década de 1960 (BOSSERT y CÓRDOBA, 2015; GORDILLO, 1999).

Su lengua presenta una tendencia a la polisíntesis, oposición verbo/nombre, marcación de núcleo en lo que respecta a la relación entre predicado y argumentos, y ausencia de marcación de caso y adposiciones. En cuanto al orden de los constituyentes, las combinaciones más utilizadas son: VS ${ }^{5}$ y PVA (cuando $P$ se expresa como pronombre libre). Los índices pronominales en los verbos presentan alineación escindida, de acuerdo a la jerarquía de persona: predominantemente nominativoacusativo cuando se trata de los participantes en el acto de habla y tripartito en la tercera persona. Una de las particularidades, dentro de la familia lingüística guaycurú, de la lengua de los Toba occidentales de la zona del río Pilcomayo Medio en Argentina reside en la codificación de una distinción entre grupo y grupo restringido en los índices pronominales verbales de primera persona no-singular sujeto. El patrón morfológico de esta escisión es el mismo que se observa en la codificación de la distinción entre inclusivo/exclusivo en los índices pronominales de lenguas de las familias mataco-mataguaya (excepto chorote), tupí-guaraní (especialmente tapiete) y zamuco (únicamente en

\footnotetext{
se los denomina "partidos" y "comunas", respectivamente.

${ }^{5} \mathrm{~S}=$ argumento único de la cláusula intransitiva; $\mathrm{A}=$ argumento más parecido al agente de cláusula transitiva; $\mathrm{P}=$ argumento más parecido al paciente de cláusula transitiva (COMRIE, 1978, p. 331).
} 
chamacoco). La variedad de toba analizada cuenta, además, con afijos verbales que codifican locación y dirección, algunos de los cuales se comportan como aplicativos. Los verbos carecen de expresión morfológica de tiempo. Esta categoría es expresada a través de los demostrativos y adverbios temporales. El aspecto gramatical es codificado por medio de afijos verbales. A diferencia de las variedades de toba de la provincia de Chaco, presenta una distinción en los demostrativos en función de la referencia exofórica o endofórica (CARPIO, 2012 y 2014).

El corpus lingüístico y etnográfico analizado en este trabajo fue recolectado en sucesivos trabajos de campo que realizamos en la comunidad de Vaca Perdida y La Mocha (Departamento Bermejo, Formosa, Argentina).

\section{El sol como indicador de los momentos del día en otros pueblos indígenas sudamericanos}

El sol es uno de los más importantes indicadores de los momentos del día desde la antigüedad hasta nuestros días. Utilizar directamente la posición del sol en el cielo para indicar el lapso del día al que se refieren también es común entre otros pueblos indígenas. Por ejemplo, en el área andina, este tema ha sido estudiado en Misminay (Cusco, Perú). Allí el sol se utiliza para coordinar actividades y compromisos en el futuro y para describir el instante en el que algo sucedió en el pasado. Según la descripción que realizan, se entiende que el sol "se desplaza a través del firmamento como un reloj: nace en el este cerca de las 6:00 am, se encuentra directamente encima al mediodía y se interna en el mar (mar cósmico) en el oeste, alrededor de las 6:00 pm" (URTON, 2005, p. 72). Es decir, la hora se relaciona con la posición del sol en el firmamento y el autor destaca que dicha posición se indica no solo marcando un lugar en una línea imaginaria que va de este a oeste sino también respetando, aproximadamente, su declinación norte/sur. A su vez, el estudio 
realizado por Floyd (2016) muestra cómo la referencia temporal es codificada entre los Nheengatú de la Amazonía (área del Río Negro, Brasil, Colombia y Venezuela) de manera multimodal, es decir mediante estrategias auditivas y visuales/corporales, señalando una posición en el firmamento sobre el eje este-oeste. Ambas modalidades son portadoras de elementos lingüísticos significativos. El autor se explaya sobre otros estudios y etnografías en los que se describe cómo se utiliza la misma modalidad visual para referirse al transcurso del día. Esta es también una de las estrategias de los Toba para demarcar los diversos instantes del ciclo diurno A su vez, en las tierras bajas sudamericanas, los Kanamari utilizan el sol como marcador temporal y espacial (REESINK, 1989, p. 122). Lo mismo sucede con los Guahibo del Orinoco, quienes utilizan la posición del sol en el firmamento para determinar un momento específico del ciclo diurno (BAQUERO, 1989, p. 135-136). Los Bororó también utilizaban el sol para especificar momentos del día y eso lo hacían haciendo un gesto con su mano, con lo cual indicaban la posición que ocupaba el sol en el instante que por algún motivo se quiere especificar (ALBISETTI y VENTURELLI, 1962, p. 295 apud PEDROZA LIMA et al., 2013, p. 117).

En el Chaco central, en los alrededores de donde actualmente habitan los Toba, exploradores y etnógrafos han brindado información sobre algunos objetos celestes. Respecto del sol y su relación con el paso del tiempo citamos una escueta referencia brindada por Storm (1892, p. 123): "El sol les sirve como reloj". Nordenskiöld (2002 [1912], p. 27) relata que, entre los Chorotes, al "reloj" de sol lo llaman: kílekíe y, según el explorador sueco, habían visto cómo lo utilizaban los blancos. En Chorote también se observa el recurso al lexema "sol" para referirse al reloj: en la variedad yojwáha, el nombre ki'lje? "sol" ocurre en ki'lje-'kje? "reloj" (GERZENSTEIN, 1978, p. 81, 83) -este nombre es segmentado por Carol (201 1, p. 434) como kiljé-kje? "sol-[3Pos]herramienta"- y en las variedades yowúwa y manxúj el lexema ki'laj "sol" también forma parte de la expresión para referirse al reloj ki'laj-xi'kje? (yowúwa) y ki'laj 'toja "reloj" 
(manxúj) (GERZENSTEIN, 1983, p. 218, 221). Del mismo modo, en wichí ocurre la palabra sol en la expresión que codifica al reloj pulsera al cual Ilaman kaijwala(s), mientras que el sol, (del mismo modo que el día y la luz) es denominado ijwala (MONTANI, 2017, p. 511). Respecto de los Pilagá, Palavecino (1933, p. 8) expresa que el paso de los días lo registraban señalando cuantas veces pasaría el sol por el cielo hasta llegar al punto que querían indicar, precisando de la misma forma la hora de llegada. Según López (2009, p. 259), entre los Mocoví también se señalan los momentos del día tomando como guía la posición del sol y para marcarlos se hace un gesto hacia el cielo. Utilizaban, además, diversas variantes de un gnomon para marcar los instantes de la jornada; este instrumento podía llegar a ser una rama pequeña. Lo mismo sucede hoy día entre los Toba cuando se refieren a un momento del día en particular. Como se verá posteriormente, indican con el brazo extendido hacia el cielo el lugar en el que estará el sol en ese instante que por algún motivo quieren resaltar. Uno de los colaboradores comentaba: "los ancianos de esa palabra estaban diciendo como: ese reloj es como un sol. Ese reloj es como un sol, a eso se refiere ahéwa likíri".

\section{Nombres que se refieren al reloj en toba del oeste de Formosa}

En toba del oeste de Formosa, es posible referirse al reloj a través de nombres derivados: lowágahe "su reloj" (lit. "entidad femenina relacionada con la mano") -a partir de la base nominal -owáq "mano"- (1) e yikatená "mi reloj", "revista", "computadora" (lit. "mi objeto femenino que miro")-a partir del verbo -ikatén "mirar"- (2), o de la combinación de los nombres ahéwa "sol" y likí?i "su imagen", "foto", "espíritu"' -ahéwa

\footnotetext{
${ }^{6}$ Nikíîi en toba del oeste de Formosa es pronunciado como nki i en las variedades orientales. Tola (2012, p. 154, 160-162) sostiene que el nkiri "persona potencial", "ser sin cuerpo" constituye uno de los componentes que los qom asocian a la persona junto a lpaqal "sombra", napaGayaGa "calor corporal" -que deriva del movimiento del cuerpo y de la circulación de la sangre y está asociado a la existencia de un nki $i i$ corporizado-, las facultades corporales como los pensamientos y los sentimientos, y nlonaGat "nombre", el cual guarda una relación metonímica con la persona y visibiliza el lazo social de cada individuo con su grupo. Según Wright (2015, p. 34-35, 38), el lki'i "su alma-imagen" es el componente de los seres humanos
} 
likípi “reloj” (lit. "imagen del sol")- (3). Respecto del likíri del sol o ahéwa, los testimonios registrados mediante trabajo de campo coinciden en que es como una imagen o un "dibujo" que hace el sol, lo que en nuestros términos remite a la sombra que proyecta todo lo que es iluminado por él. La imagen que proyecta el sol o likípi puede ser percibida por todas las personas, siendo ese uno de los factores que les permite saber en qué momento del día se está. En este contexto preciso, el likípi del sol parece asociarse a la idea de que el reloj hace las veces del sol, en tanto refleja sus movimientos y los movimientos que haría cualquier reloj de sol. De ahí que expresaran: "el reloj es como un sol" o "el reloj sigue al sol".

Los tres lexemas registrados para referirse al reloj son asignados al género femenino, como puede apreciarse a través de la morfología de género en los nombres derivados (1)-(2) y en la concordancia con los demostrativos a través del prefijo ha- en (1)-(3).

\title{
(1) ha-ñíp-me l-owága-he- $\varnothing$
}

F-DSE-ENDOF ${ }^{7}$ 3POS.INAL-mano-REL-F

"Su reloj" (lit. "entidad femenina relacionada con la mano")

(2) wo? ha-ñí? y-ikaten-á

EXIST F-DSE 1POS.INAL-mirar-NOM.PAC.F

"Tengo un/a reloj", "revista", "computadora" (lit. "mi objeto femenino que miro")'
(3) ha-ñí? ahéwa nogotolé-k
F-DSE SOl 3POS.INAL-imagen 3II-despertar n-oqtogón díp-me I-ikípi DACOS- ENDOF niña-MASC
"El reloj despierta al niño"

\begin{abstract}
que sale durante los sueños cuando la persona está dormida, guarda la conciencia del individuo y "puede trasladarse por diferentes lugares del cosmos". El alcance de las experiencias oníricas depende del estatus de poder respectivo de quien sueña. La capacidad de "viajar" o "caminar" en sueños y de controlar a voluntad su lki' $i$ es propia de los pi'oGonaq o shamanes, acompañados de entidades poderosas (lowanek "su mandado", "vigilante"). Idoyaga Molina (1995) ha explorado este tema entre los Pilagá describiendo al $k i$ ' $i$ en conjunto con el paqál. Según la autora, todos los individuos pueden percibir el ki'i en tanto imagen refleja, puesto que si se hiciera referencia al paqál, eso quedaría bajo la órbita de dominio de los pyogonáq. 7 Abreviaturas: 1, 3= primera y tercera persona; ALIE=alienable; CAUS=causativo; DACOS=demostrativo 'acostado'; DAL=demostrativo ‘alejándose'; DPROX=demostrativo 'próximo'; DSE=demostrativo 'sentado'; ENDOF=endofórico; EXIST=presentativo existencial; $\mathrm{F}=$ femenino; I, II=índice pronominal tipo I y II; $\mathrm{INAL}=$ inalienable; $\mathrm{MASC}=$ masculino; $\mathrm{NOM=nominalizador;} \quad \mathrm{PAC}=$ paciente; $\quad \mathrm{PL}=$ plural; $\quad \mathrm{POS}=$ poseedor; REL=nominalizador 'relativo a'.
\end{abstract}


Analizaremos la construcción ahéwa likípi junto a otras construcciones de tipo [nombre $+I$-nombre] ${ }^{8}$, las cuales presentan un comportamiento fonológico y morfológico que las ubican entre los compuestos y las frases.

Los compuestos, derivados y frases nominales constituidas por dos nombres yuxtapuestos no siempre son claramente distinguibles puesto que los tres son funcionalmente equivalentes, es decir permiten expandir el inventario léxico de la lengua. Como sostienen Givón (2001, p. 68) y Booij $(2007$, p. 82,85$)$, esta proximidad funcional en la sincronía se correlaciona con el hecho de que las combinaciones de palabras en frases pueden funcionar como precursoras de la composición y ésta de la derivación. No obstante, la composición, las frases constituidas por dos lexemas yuxtapuestos y los derivados pueden ser distinguidos a partir de una serie de rasgos formales.

La principal diferencia entre los compuestos y los derivados reside en que, como plantea Booij (2007, p. 85-86), los constituyentes de los primeros son lexemas, mientras que los segundos implican la presencia de afijos, es decir, uno de sus constituyentes no ocurre como lexema en la sincronía. Este criterio no siempre es fácilmente aplicable porque puede suceder que un mismo morfema ocurra, en cierto corte sincrónico, como afijo y aún corresponda a un lexema. Estos morfemas son denominados afijoides (affixoids).

Siguiendo lo planteado por Haspelmath (2002, p. 155-161) y Booij (2007, p. 82), es posible distinguir los compuestos de las frases constituidas por dos lexemas yuxtapuestos a partir de una serie de rasgos fonológicos, morfológicos y sintácticos. En los tres tipos de rasgos considerados los compuestos se caracterizan por una mayor cohesión que las frases. En términos fonológicos, los compuestos pueden poseer, por ejemplo, un patrón acentual particular o implicar cambios

\footnotetext{
${ }^{8}$ Esta estructura morfosintáctica es recurrente en nombres compuestos de plantas y animales (CÚNEO 2013, p. 244).
} 
morfofonológicos a nivel de los segmentos (mayor cohesión fonológica). Los procesos morfológicos afectan al compuesto como un todo y los constituyentes internos de los compuestos son inseparables, es decir no pueden ocurrir modificadores nominales entre los componentes de los mismos (mayor cohesión morfológica). Además, el nombre dependiente de un compuesto no puede ser reemplazado por un pronombre anafórico y sus constituyentes no pueden ser dislocados a posición inicial de cláusula ni pueden ser elididos en coordinación (mayor cohesión sintáctica).

La yuxtaposición de frases nominales para expresar una relación de posesión y/o de tipo meronímica (parte/todo) en lo que respecta a la estructura morfosintáctica y los rasgos semánticos ha sido analizada en las variedades orientales de toba, especialmente de la provincia de Chaco, por Klein (2000, p. 85), Censabella (2002, p. 277) y Messineo (2003, p. 133), y con especial referencia al léxico etnobotánico por Cúneo (2013, p. 241-247) y Martínez y Cúneo (2009).

Siguiendo lo propuesto por Booij (2007, p. 83), proponemos considerar las combinaciones de [nombre $+l$-nombre], entre las que se encuentra ahéwa likíii "reloj", como construcciones idiomáticas (constructional idioms). Es decir, patrones sintácticos fijos que permiten expandir el conjunto de unidades léxicas de la lengua y en los que algunas posiciones son ocupadas por morfemas o palabras específicos y otras por todo tipo de palabras de la categoría correcta. En los ejemplos analizados, el constituyente fijo es el prefijo de posesión de tercera persona l- en el nombre que se ubica a la derecha y los constituyentes variables son nombres cuyo repertorio es más amplio en la primera posición respecto de la segunda. Esta última posición se restringe a nombres compatibles con el paradigma de posesión inalienable. ${ }^{9}$

En la variedad de toba analizada, las construcciones [nombre $+I$ nombre] se acercan a los compuestos porque:

\footnotetext{
${ }^{9}$ Carpio (2012, p. 44-96) presenta un análisis integral de las frases nominales en toba del oeste de Formosa.
} 
i. presentan alternancias morfofonológicas entre los nombres combinados idénticas a las que ocurren cuando se sufijan morfemas a dichos nombres (4).

En la combinación del nombre pegáq "caballo" (4a) con el nombre inalienable likí?i "su imagen", "foto", "espíritu" (4b) utilizada para referirse a la "bicicleta" (lit. "imagen o foto del caballo") (4c) se produce la misma variación morfofonológica en el nombre que se ubica a la izquierda (pegáq "caballo") que cuando, por ejemplo, se pluraliza a través del sufijo -di nombres cuyo fonema final también es /q/ (5b). Es decir, la consonante oclusiva uvular sorda (/q/) final del nombre se realiza como sonora y se adiciona la vocal /a/.

(4) a. ñi? pegáq
DSE caballo

"Caballo"

b. hen l-ikí?i

DPROX 3POS.INAL-imagen

"Su foto", "alma", "espíritu"

c. ha-ñíp-me pégaca l-ikípi

F-DSE-ENDOF caballo 3POS.INAL-imagen

"Bicicleta" (lit. "imagen o foto del caballo")

(5) a. kétaq

"Cabra"

b. kétaca-di

chiva-PL

"Cabras"

ii. ocurren junto a un único demostrativo antepuesto a ambos nombres que concuerda en género con uno de ellos. Si al menos uno de los nombres combinados es asignado al género femenino, generalmente, el nombre compuesto concuerda en dicho género con el demostrativo (6).

(6) a. ha-ñíp-me ahéwa y-ipogóy-agat aléwa

F-DSE-ENDOF sol 3I-reventarse-CAUS DPROX-ENDOF tierra "El sol hizo partir la tierra" 


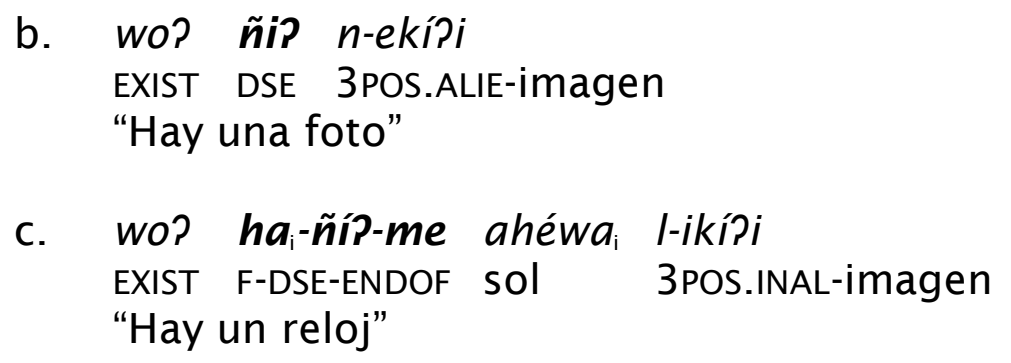

iii. poseen una única flexión de número que afecta a todo el conjunto.

En (7) el morfema de número -I se sufija al nombre likípi 'su imagen, foto, espíritu' y pluraliza a toda la construcción ahéwa likí?i, mientras que el nombre ahéwa permanece invariante.
(7) h-awána-lo há<a>?-me lamá?i ahéwa l-ikípi-I
1l-tener, encontrar-PL DNP $\quad$ PL>-ENDOF CUANT sol
3POS.INAL-imagen-PL
qápli
ADV
"Yo tenía varios relojes, antes"

iv. el nombre que se ubica a la izquierda puede recibir flexión de posesión, la cual posee un alcance sobre todo el compuesto nominal.

En (8) el morfema posesivo ñ- 'primera persona singular poseedor' ocurre junto a ahéwa -nombre no poseído cuando ocurre solo- con el fin de expresar que el referente de la construcción idiomática ahéwa likípi pertenece al hablante.
(8) han $\tilde{\boldsymbol{n}}$-ahéwa l-ikíri
DPROX.F 1 POS.ALIE-SOI 3POS.INAL-imagen
"Mi reloj"

Sin embargo, en el corpus analizado, se registraron construcciones [nombre + l-nombre] que presentan rasgos morfológicos que las aproximan a las frases. Por ejemplo, cuando se combinan los nombres nogóp "agua" (9a) y láyt "su contenedor" (9b), si bien se observa cohesión morfológica puesto que ambos son modificados por un único 
demostrativo que concuerda en género con el nombre 'layt "su contenedor" (9c), se asemejan a una frase puesto que poseen autonomía morfológica, cada uno puede recibir flexión de número (9d).

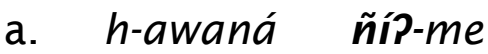
nogóp
1I-encontrar
DSE-ENDOF
agua
"Encontré agua"
b. h-awaná háp-me l-áyt
1I-encontrar DAL.F-ENDOF 3POS.INAL-contenedor
"Encontré el camión (contenedor)"
c. h-ekatén ha $a_{\mathrm{i}}$-̃̃íp-me nogóp l-áyt $t_{\mathrm{i}}$
1I-mirar F-DSE-ENDOF agua 3POS.INAL-contenedor
"Miré la represa"
d. h-ekaté<?>n noqop-í l-éte
1I-mirar<PL> agua.PL-PL 3POS.INAL-contenedor.PL
"Miré las represas"

A su vez, en construcciones [nombre + l-nombre] se observó sincrónicamente la relación diacrónica que existe entre composición y derivación al comparar, por ejemplo, los usos del nombre lagalá? "su lugar de apoyo" y del sufijo -gala? "lugar de apoyo". El nombre lagalá? ocurre: a. junto a otro nombre, como en $(10 a)$, comportándose a la manera de las construcciones idiomáticas descriptas anteriormente, y $b$. no acentuado, sin el prefijo de posesión $I$-, funcionando como un sufijo derivativo (10b).
(10) a.
hó?-me y-ahépl l-agaláp
DAL-ENDOF 1 POS.INAL-bastón 3POS.INAL-lugar de apoyo "Mi soporte" (Lit. "Mi lugar de apoyo del bastón")
b. hóp-me y-a'pya?-Gala?
DAL-ENDOF 1 POS.INAL-PIe-LUGAR DE APOYO
"Mi escalera"

\section{Estrategias para leer los distintos momentos del día entre los Toba}


A partir del análisis lingüístico propuesto, podemos apreciar que la construcción idiomática con la que se refiere al reloj en toba pertenece al género femenino $y$, por otra parte, al hacer referencia al sol, da cuenta de una forma específica de marcar el paso de la jornada entre los antiguos y algunos de los actuales Toba. Hombres mayores y otros ancianos explicaban cómo se refería a los distintos momentos del día y a partir de qué pautas lo construían. Una de las formas de hacerlo es por medio de lo que en astronomía académica se llama día solar verdadero ${ }^{10}$, que "resulta del intervalo de tiempo transcurrido entre dos pasajes sucesivos del sol verdadero por el meridiano del lugar" (FEINSTEIN, 1969, p. 44). Este tipo de medición de tiempo es el que brinda un reloj de sol. Un tipo de reloj de sol que es bastante sencillo de realizar es aquel donde se planta una varilla vertical sobre la tierra, siendo éste el formato que concuerda con los relatos y experiencias narradas por los Toba." Según la ubicación de la sombra que proyecta la varilla en el suelo, puede leerse el momento del día en el que se está. Esta forma de señalar el tiempo no es uniforme o similar durante todo el ciclo anual. Como la posición del sol en la esfera celeste varía a medida que transcurre el año, la sombra proyectada varía con el transcurso de los meses. Por ejemplo, no está en la misma posición un mediodía del mes de septiembre que un mediodía del mes de junio. ${ }^{12}$ Así nos explicaban que "cuando hay tiempo de algarroba", hacia el mes de diciembre, durante los momentos de calor del ciclo anual el sol va "bien derechito", "bien en el medio de donde vivimos": chimqáchiyiñi. En tanto que en la época de frío, cuando cae la helada, consideran que el sol "se corre": nichík. Por otro lado, tal como lo advierte

\footnotetext{
${ }^{10}$ Otra forma de tiempo empleada puede ser el día solar medio, que es el promedio obtenido de los días solares verdaderos. Usando el día solar medio se define el tiempo civil, aquel que llevamos en nuestros relojes (FEINSTEIN, 1969).

${ }^{11}$ Otra de las formas de realizarlo es colocar una varilla inclinada con respecto al horizonte en un ángulo igual a la latitud del lugar, lo cual no ha sido registrado entre los Toba.

${ }_{12}$ A su vez, se ha señalado que puede leerse en qué época del año están por la longitud de la sombra que presente el palo o la varilla del "reloj" de sol que utilizaban. Sin embargo, para indagar sobre este aspecto, a esta referencia es indispensable sumarle muchas otras y desbordarían el tema que se trata en este trabajo. Para indagar sobre otros indicadores que marcan o anuncian los períodos del ciclo anual, Cf. Arenas (2003), Arenas y Porini (2009), y Gómez (2012).
} 
la mitología toba, durante el verano el sol parece moverse más lentamente, en tanto que, durante el invierno, lo hace mucho más rápido, situación que también repercute en el movimiento de la sombra que proyecta. ${ }^{13}$ A su vez, durante el verano el sol es una mujer gorda que va lentamente por el cielo, en tanto que durante la época de frío es una joven que transita rápidamente (MÉTRAUX, 1946a, p. 19). Esta es una de las explicaciones acerca de la duración variable del día durante el ciclo anual.

Sin embargo, durante las charlas que hemos tenido con los Toba sobre el cielo, el sol no suele ser un tema sobre el que se explayen mucho, sobre todo si se lo compara con algunos objetos celestes que son significativos marcadores temporales como por ejemplo Awógoyk "luna" (GÓMEZ, 2010), Dapichí y Qarkotél. Estos dos últimos asterismos son representados en el cielo por las Pléyades y por El Cinturón de Orión, respectivamente (GÓMEZ, 2012). Es recurrente la mención de que el sol es más poderoso que la luna, situación que coincide con la mitología que puede leerse sobre este astro (WILBERT y SIMONEAU, 1982, p. 27-31, 95, 101). A su vez, han destacado que los pyogonáq no pueden entrar en el sol (ahéwa) porque es muy fuerte y se han referido a él como una figura femenina. Esto último puede apreciarse también en la morfosintaxis de la lengua a través de la concordancia de género femenino con el demostrativo del nombre ahéwa "sol" (6a).

Actualmente, entre los Toba, se han rastreado varios métodos para señalar los momentos en que dividen la jornada, los cuales consisten en la utilización de: a. el brazo, b. un gnomon, c. "sombras", y d. otros objetos celestes, además del sol, animales y teléfonos celulares.

a. Utilización del brazo

\footnotetext{
${ }^{13}$ Ambos fenómenos se deben a que el sol se desplaza sobre la eclíptica, la cual por estar inclinada $23^{\circ} 27^{\prime}$ respecto del ecuador terrestre determina que su proyección sobre el mismo sea diferente a lo largo del año. Además, "la trayectoria de la tierra alrededor del sol es una elipse, lo que implica que su velocidad de movimiento sea variable" (FEINSTEIN, 1969, p. 50).
} 
Una de las estrategias utilizadas recurre directamente a la posición del sol en el firmamento. Cuando no se decía la hora en español, uno de los colaboradores explicaba que para indicar un momento de la jornada los ancianos decían:

Gá?me ná?ñá... Y para completar la palabra: ahéwa... gá?me ná?ña hañípme ahéwa (...) Entonces, ¿a qué hora, a qué distancia [viene]? Entonces ya uno puede saber, calcula más o menos...Pero no se sabe qué hora está pero calcula... O sea, el sol viene de... Cuando sale el sol entonces dice, si un anciano dice: ná?ña, el sol sale así.

La escena narrada se completaba con el movimiento de su brazo extendido, el cual señalaba hacia una posición que apuntaba a cierta distancia del piso y se dirigía a la bóveda celeste. Lo enunciado y la posición que se marca por medio del brazo, son formas de señalar inseparables, una no tiene sentido sin la otra. Hoy en día también se señala con el brazo totalmente extendido directamente al cielo la ubicación que el astro debería tener según el momento al que se hace referencia. De esta forma, se da cuenta del momento del día del que se habla con bastante precisión, también esa puede ser la forma de pautar el momento fijado para llevar a cabo un encuentro. Junto a otros indicios, que se referirán más adelante, el sol es un claro indicador del transcurso del día, y su asociación con el reloj es inmediata, es así que explicó uno de nuestros consultantes que "el reloj está siguiendo ahéwa". El movimiento de las agujas del reloj es asociado al que realiza el brazo extendido hacia el cielo indicando la ubicación del sol en algún instante.

\section{b. Gnomon}

La utilización de un gnomon se daba sobre todo en el monte. Con el fin de saber dónde estaba el sol buscaban "un campito" y utilizaban un palo clavado en la tierra. Uno de los entrevistados nombró a este 
instrumento como lanagánaqate [laná:naqate] ${ }^{14}$, es decir la herramienta que se utiliza para sembrar, asignada al género femenino (11).

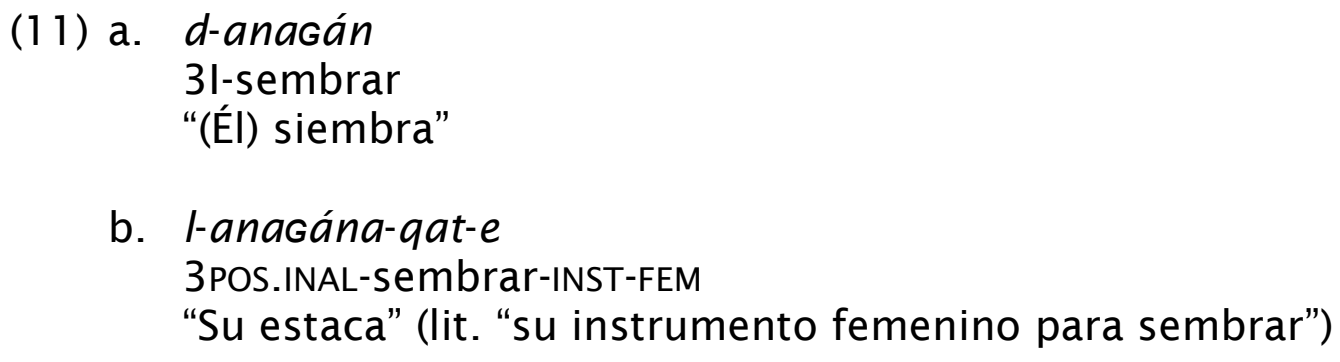

Si tenemos en cuenta que se clava en el suelo, no sería extraño que también se utilizara con este fin el elemento usado para sembrar. Uno de nuestros entrevistados explicaba que mediante ese instrumento "la sombra va marcando para que el hombre pueda saber a qué hora... a qué distancia, vamos a decir, donde está el sol, si ya es alto o todavía bajo, es decir tempranito, si ya está un poco alto. Entonces marca esa estaca como una señal". Según la sombra que diera el palo sobre el suelo corroboraban si regresaban al poblado. Uno de los entrevistados indicó que la varilla clavada en el suelo también podía ser utilizada en la casa.

\section{c. "Sombras"}

Además del gnomon, también indicaron que se podía saber el momento del día gracias a la sombra que proyecta cualquier parte de la casa sobre el suelo, puesto que se puede leer la sombra de cualquier cosa. A su vez, la sombra de una persona podía ser utilizada y dar la pauta del momento del día. Todas estas estrategias, este conocimiento fundamental para el desarrollo de algunas de sus actividades, forma parte del repertorio de saberes que catalogan como: "los estudios de los ancianos"15.

\footnotetext{
${ }^{14}$ En este tipo de derivados nominales, es muy común que, en lugar de pronunciar los segmentos agan, se alargue el núcleo vocálico y se omita la consonante oclusiva uvular sonora $[a: n]$.

${ }^{15}$ Este conocimiento ha sido trasmitido de generación en generación de forma oral por los más ancianos, remitiendo mayormente a los saberes que son identificados como anteriores a la sedentarización, previos a la llegada y establecimiento entre ellos de los misioneros anglicanos.
} 
d. Otros objetos celestes, animales y teléfonos celulares

El sol no era ni es la única guía. El lucero o Venus es otro importante marcador del comienzo y fin de la jornada. La aparición de Níchigi, el lucero o Venus matutina, apunta que ya viene el día y es hora de partir hacia al monte. Inclusive cuando estaban trabajando en los ingenios, la aparición de Níchigi seguía siendo una referencia. Un colaborador contaba que, antes de escuchar la campana que tocaba el capataz para ir a trabajar, ellos ya estaban despiertos porque "la estrella grande" ya indicaba que venía el día. Por su parte, la aparición de Potaqáe, representada en el cielo por Venus vespertina, señalaba que es necesario regresar del monte, volver al hogar. Como explicó un anciano durante uno de los primeros trabajos de campo: "Los viejos saben más de las estrellas aunque no tienen reloj", lo que ya demuestra una asociación entre algunos objetos celestes y el transcurso de la jornada. Durante la noche se hace referencia a la luna, la vía láctea y según el instante del ciclo anual en el que están, se puede saber el momento de la noche por la posición de otros asterismos que identifican en el cielo.

Sin embargo, observar el cielo no es el único camino que guía respecto del devenir diurno y nocturno. Las aves se suman a este cúmulo de indicadores y los loros, por ejemplo, serían uno de ellos. Según Gordillo (2005, p. 48), los loros -elé? (Amazona aestiva)- daban la pauta del mediodía, en tanto que de acuerdo con Arenas y Porini $(2009$, p. 52$)$ cuando los loros regresaban a sus nidos eran una clara señal del crepúsculo. Por su parte, a ahéwa lalóp ${ }^{16}$ (Pyrocephalus rubinus; coloquial: brasita de fuego) se lo asocial al verano y anuncia las horas con su canto, sobre todo se hace referencia que canta al amanecer: "es como un despertador" (ARENAS y PORINI, 2009, p. 213). Sobre ahéwa laló? un anciano ya fallecido explicaba que a esa ave se la veía durante la época de calor apenas amanecía y siendo tan roja como es el sol cuando

\footnotetext{
${ }^{16}$ Ahéwa laló? literalmente significa 'el animal doméstico del sol' y en términos morfosintácticos se trata de una construcción idiomática del mismo tipo que ahéwa li 'ki?i 'reloj'.
} 
amanece. Del mismo modo, palálogo (Xolmis irupero) también anunciaría el día pero durante la época fría del ciclo anual, puesto que su aparición se enlaza a este período. Otra de las aves con estas características de anunciación es nehógoe (Tyrannus melancholicus) la cual, según lo relevado por Arenas y Porini (2009, p. 216), si canta es porque se acerca el ocaso: "si una persona está perdida o se le hizo tarde en el monte, al escucharlo le produce angustia y temor". Según algunos testimonios, su nombre recuerda a la mujer caníbal (WILBERT y SIMONEAU, 1982, p. 312 314). Los gallos también fueron nombrados como anunciadores del día, aunque este aspecto parece más ligado al pasado reciente de los Toba. Este tipo de "señales" tiene la característica de servir de apoyo para poder percibir algunos momentos de la jornada en días nublados y también durante la noche. Por ejemplo, se sabe por el canto de la perdiz que ya es la medianoche. No sólo los pájaros generan indicadores de este tipo, también lo hacen otros animales como wagayagá (Lycalopex gymnocerus), el zorro, que se lo puede escuchar cuando ya pasó la medianoche; el mismo lapso nocturno es anunciado por la lechuza o kidikík (Asio clamator, Bubo virginianus) (ARENAS y PORINI, 2009, p. 53).

El principal objetivo que se enlazaba a la utilización de un gnomon en todas las conversaciones era ayudar a señalar el regreso desde el monte hacia el poblado. Lo más importante era que no se hiciera de noche mientras se estaba en el monte, situación que coincide con la angustia que causa el canto de nehógoe (Tyrannus melancholicus); al escucharlo se sabe que hay que irse, pues se está acercando el crepúsculo. Los indicadores citados más recurrentemente eran aquellos que señalaban el momento de dar inicio o fin a las actividades cotidianas que se realizaban, lo que implicaba la salida de la casa o el regreso a ella ya sea por medio de la lectura de la altura del sol o por las sombras o dibujos que proyectara mediante la utilización cualquier elemento o gnomon. Hoy en día, los más jóvenes llevan también al monte el celular, no para comunicarse (cosa que allí todavía es imposible) sino para saber la hora: 
Las mujeres ancianas digamos que hay un poco de ancianas que sí, que todavía siguen eso [guiarse por el sol, su sombra y/o el canto de diversos animales]. Pero como yo digo, las jóvenes, las adolescentes... como yo, yo ya sé la hora... Y sí, digamos hay una diferencia pequeña, pequeñitos cambios que nosotros ya manejamos. Ahora ya no es ni la hora... ni reloj sino por celular. Guiamos por la hora viste, que ya miramos. Por eso por ahí nosotros llevamos al monte el celular, ¿por qué?: porque la hora.

\section{Lapsos de la jornada: nombres, actividades y significados}

Cuando se inquiere sobre cómo dividen el día o noló?, algunos colaboradores expresan que se dice nálañi cuando es muy temprano y son los instantes en que todavía está oscuro y no ha salido el sol. ${ }^{17}$ Hay mayoritaria coincidencia al expresar que al amanecer se lo nombra como yogoñí, en tanto que dicen néteta para señalar la mañana. Para referirse al mediodía dicen noló? layñí, háwit ['haßit] para la tarde y píyaq para la noche, mientras que a la medianoche se la nombra como píyaq lawél ${ }^{18} \mathrm{y}$ también píyaq layñí. Noló? layñí "mediodía”, píyaq layñí "medianoche” y píyaq lawél "medianoche" son otros ejemplos de construcciones idiomáticas del mismo tipo que ahéwa likípi 'reloj' compuestas por los nombres noló? "día” y píyaq "noche” junto al nombre inalienable layñí "su centro" y lawél "su estómago". Cuando indicaban un momento específico del día, el marcador más inmediato era colocar el brazo en alto señalando hacia el cielo para indicar en qué posición debía estar el sol en ese momento. Actualmente, junto con el reloj y el celular, esta última forma de indicar instantes $u$ horas aproximadas suele ser general.

El momento marcado como noló? layñí es de los más fáciles de identificar, pues es cuando el sol está "justo en el medio" y la sombra que genera es, según describen, "bien derechita”. Ahora bien, fuera de los

\footnotetext{
17 Antiguamente el momento anterior al amanecer también era importante para las incursiones guerreras (MÉTRAUX, 1946b, p. 314; ARNOTT, 1934).

${ }^{18}$ Lawél significa "su estómago" y es una de las partes del cuerpo utilizada para especificar la posición de una entidad, específicamente indica que se encuentra ubicada en el centro.
} 
indicadores relacionados a los animales y otros objetos celestes, en principio, no parece inmediatamente claro cómo marcar ese lapso nocturno llamado píyaq lawél o piyáq layñí y sobre todo el porqué de su nombre. Sin embargo, si uno presta atención a la forma en la que era descripta la tierra y el cielo según "el estudio de los ancianos" los términos parecen ser mucho más evidentes. Cuando al comienzo de nuestra investigación nos describían en forma general a la tierra, el cielo y el trayecto de ciertos objetos celestes, contaban que por debajo de la tierra habitada por los hombres hay otra tierra y que cuando aquí es de noche, el sol ilumina debajo de la tierra. De esta forma uno de los ancianos enunciaba: "el sol alumbra a la tierra que está debajo de la que vivimos nosotros y cuando eso sucede aquí está oscuro"; descripción que ya ha sido registrada en la mitología toba (LEHMANN-NITSCHE, 1924/25, p. 182; WILBERT y SIMONEAU, 1982, p. 30), y pilagá (IDOYAGA MOLINA, 1989, p. 21 ; TOMASINI, 1976, p. 83). Del mismo modo, otro hombre toba que domina muy bien el español intentaba explicarnos los movimientos del sol en el cielo y como éste marcaba los instantes en los que puede dividirse el día. Uno debe imaginarse una circunferencia que atraviesa el cielo, mientras expresaba esta idea marcaba con su brazo hacia el cielo del horizonte este al horizonte oeste y explicaba que ahí uno puede entender cómo durante el mediodía el sol está justo en el medio de esa circunferencia imaginaria:

Bien derechito, ahí es noló? layñí [...]. Noló? layñí. Es decir, bien así [hace gesto con el brazo extendido indicando hacia el cielo siguiendo el eje de su cuerpo] [...] Bien derecho. Como... hacé un círculo redondo así, bien en el medio, ahí está el sol.

Siguiendo esta descripción no es difícil imaginar que cuando el sol realiza el mismo trayecto por debajo de la tierra, lo hace del mismo modo que arriba y al estar en el medio pero por debajo de la tierra sería el momento en el que se da piyáq layñi o píyaq lawél en la superficie terrestre. De hecho, mientras repasábamos los movimientos del sol, el 
mismo hombre explicaba que cuando era noló? layñí, no significaba que no había sombra, sino que la sombra estaba derecha y ratificaba que eso sucedía porque el sol estaba: "bien derecho". Para completar la descripción expresó: "igual que cuando decimos de noche: píyaq, píyaq layñí. Píyaq layñí quiere decir a las doce de la noche". En este contexto, lo expresado nos lleva a pensar que la reflexión sigue con la misma lógica de los ancianos, el sol sigue por debajo de la tierra e ilumina ese espacio de la misma forma que cuando hace su recorrido por "arriba", con lo cual allí el sol también estaría bien derechito, pero en este caso por debajo de la tierra habitada por nosotros, siendo este momento: píyaq layñí. Esta construcción no tiene como fin marcar con precisión la hora exacta en que se da la medianoche, sino que así se establece un lapso más de la noche, que al igual que noló? layñí coincide aproximadamente con la hora marcada por los criollos, pero manteniendo el orden o la cosmovisión heredada de los ancianos. Hay que subrayar que hasta ahora no han marcado la noche indicando el trayecto del sol con el brazo hacia abajo. No obstante, las ideas expresadas tienen coherencia con su cosmovisión, específicamente retoman aquello del trayecto que hace el sol por debajo de la tierra cuando aquí es de noche, mostrando una suerte de juego de espejos entre los movimientos de sol por debajo y por encima de la tierra. Por otro lado, esa semicircunferencia que marcan de horizonte este al horizonte oeste y los movimientos que realizaría el sol en ella, recuerdan el trayecto que marcan las sombras de un "reloj" solar, y el movimiento que se estima hace de noche el sol replicaría el movimiento diurno. Esto último concuerda con lo que explican en los mitos: la puesta del sol por el horizonte oeste y su salida por el horizonte este luego de haber completado su trayecto por debajo de la tierra habitada por los Toba. Sin embargo, hay que destacar que fuera de la utilización del reloj o del celular, la forma práctica de guiarse en tiempo y espacio por la noche se relaciona más con los sonidos de algunos animales, los movimientos de la luna, de la Vía Láctea y de algunos asterismos. 
Por otro lado, aunque en la práctica, el momento que marca el sol en la esfera celeste como mediodía pueda no ser exactamente igual al que marca el reloj pulsera, se entiende que ambos indican lo mismo. Tampoco parece perseguirse algún tipo de simetría entre cada uno de los instantes que se enumeran. Hay que recordar que no se persigue la precisión, ni importa la asimilación total de ambas formas de marcar el mediodía o el transcurso del día en todo momento, lo importante es que sea acorde a los fines prácticos del desenvolvimiento común de cada jornada. El mediodía también podría estar más relacionado a las formas de trabajos más recientes, como el trabajo asalariado o "las changas" donde "las doce horas" marcan el momento de descanso. Sin embargo, ese instante del día se lo tenía presente también desde antes, pues en uno de los mediodías del ciclo anual (durante el solsticio de verano) ningún reloj solar, ni ningún elemento o persona proyectaría sombra ${ }^{19}$, situación que ha sido destacada por los Toba.

Los segmentos de la jornada a los que hacen referencia con asiduidad, aquellos que más surgen durante las conversaciones son los que marcan el regreso y la ida al monte; esos eran los instantes más relevantes a ser identificados por cada una de las estrategias de lectura de los momentos de la jornada presentados en $\S 5$. Existe una diferencia crucial entre ambos momentos del día. La noche y el día tienen cualidades diversas o son susceptibles de poseerlas (HUBERT, 1946, p. 312). La noche es un momento donde se está expuesto a más peligros y estar solo o perdido en el monte durante la noche para alguien que no es pyogonáq es algo, por lo menos, peligroso. Salir o permanecer fuera del ámbito doméstico o del campamento cuando está por amanecer o a la luz del día no provoca tanto miedo e incertidumbre como si se está perdido en el monte durante la noche.

\footnotetext{
${ }^{19}$ Las poblaciones rurales en las que se asientan los Toba del oeste formoseño están, aproximadamente, a $23^{\circ} 29^{\prime}$ Sur, es decir relativamente cercanos al trópico de Capricornio. Esta ubicación hace que una vez al año el sol quede en una posición en la que las sombras son casi imperceptibles, situación que ha sido destacada en algunas conversaciones que se han tenido, donde indican que el sol queda "bien derechito".
} 


\section{Consideraciones finales}

La adopción del reloj analógico se relaciona con los primeros contactos con "el blanco". De hecho, uno de los primeros registros encontrados a fines del S. XIX -citado al principio del artículo- muestra que era utilizado como collar. Por supuesto su posterior adopción también se liga a la necesidad de responder a los horarios impuestos por los criollos y por las instituciones y visitantes que muchas veces se alojan en su poblado. El conocimiento de la hora tal como es marcada por los relojes también se enlaza al trabajo asalariado: la ida a trabajar y el descanso. Quienes tuvieron un contacto más temprano y directo con ese mundo diario ligado al paso de las horas y los minutos fueron los varones de la familia, de hecho, siempre que se vea a alguien con un reloj en su muñeca seguramente será un varón adulto. Ello se explica porque fueron mayormente los varones quienes se encargaron de las relaciones tanto "diplomáticas" como laborales con la sociedad envolvente. El reloj mecánico es un elemento que ha sido adoptado por los hombres Toba, inclusive hasta el día de hoy no se ha visto a una mujer utilizar un reloj pulsera en su muñeca, por lo menos no en público. Lo que actualmente sí sucede es que ambos utilizan el celular, el cual entre los hombres es algo que puede sumarse a la utilización del reloj. Las mujeres utilizan el celular tanto para comunicarse como para saber la hora, indispensable para relacionarse actualmente con todo lo vinculado a los contactos y trabajos que se dan por fuera de la esfera doméstica. Para ambos se impuso una forma distinta de marcar los momentos del día. Sin embargo, ello propició una confluencia o un intento de amalgamar la forma de ritmar la vida diaria que heredaron de sus ancianos y la estricta forma de dividir el día de la sociedad envolvente.

En cada explicación sobre el reloj y su asociación con el sol manifestaban qué horas correspondían a cada una de las partes en la que se solía dividir y nombrar el día en el idioma toba. Del mismo modo, cuando explicaban cómo se percibía el transcurso del tiempo diurno 
utilizando al sol como referencia, el movimiento del brazo indicando hacia el cielo la posición del astro era una constante. Aquello que aquí sólo se pone en palabras adolece de una gestualidad que es inherente de la marcación de la jornada en referencia al sol. Los diversos lugares que ocupa la sombra que proyecta un palo clavado verticalmente sobre el suelo, así como las diversas posiciones que se indican con el brazo extendido hacia el cielo se refieren al lugar que ocupa u ocupará el sol; ello se enlaza al movimiento que realizan las agujas del reloj y sería uno de los motivos que explican el porqué del nombre en idioma toba: ahewa likípi. Por otra parte, el semicírculo que hace el sol por el cielo de algún punto del horizonte este hacia algún punto oeste, desde el punto de vista toba, se replicaría al pasar por debajo de la tierra, marcando el trayecto nocturno del sol. Dos momentos cualitativamente opuestos.

La inclusión del lexema para referirse al sol en una de las expresiones lingüísticas que codifican al reloj en toba del oeste de Formosa no ha sido registrada en otras variedades de toba. Sin embargo, como mostramos en $\S 2$, en lenguas de la familia mataco-mataguaya, arealmente relacionadas con el toba del oeste formoseño, también ocurre el lexema sol en la codificación del reloj. Esta distribución nos lleva a plantear la hipótesis de que la construcción idiomática ahéwa likí?i analizada constituye un rasgo arealmente condicionado tal como sucede, por ejemplo, con el patrón morfológico de codificación de la escisión de primera persona no-singular sujeto en los verbos.

Durante estas explicaciones, sobre el reloj mecánico, el "reloj" de sol y la forma de llamarlo en idioma, se destacaba el uso de todos ellos sobre todo para corroborar que se estaba en momentos específicos del día, remarcándose sobre todo dos de ellos. Por un lado, se resaltaba el instante en el que todavía está oscuro y se aproxima la salida del sol, por el otro, el momento en el que sol está por ocultarse. Ambos lapsos ritman los polos de la actividad diurna. El amanecer era marcado como el momento propicio para salir a realizar las actividades cotidianas. En tanto que el atardecer invitaba al regreso desde el monte hacia el hogar o el 
lugar donde se tuviera el campamento. Permanecer durante la noche solo, lejos del campamento o poblado y estar perdido, implica estar más expuesto a los peligros asociados a la noche y el monte. Luego del atardecer se está más protegido en el hogar que en el monte, espacio que durante la noche es propicio para el accionar de los pyogonáq. En este contexto, ahéwa likípi opera como un indicador más que ayuda a trazar momentos con cualidades diversas: el día, donde lo cotidiano tiene lugar; y la noche: espacio peligroso al que uno sólo puede acercarse en soledad sin pasar tantos peligros siendo pyogonáq. Entonces, cuando el sol sigue su trayecto nocturno por debajo de la tierra habitada por los Toba y arriba es de noche, acontecen los instantes más peligrosos.

Aunque tanto el mediodía como la medianoche resultaran una imposición de la forma de marcar ambos momentos por la sociedad envolvente, lo interesante es que esto se entreteje con la forma de entender el trayecto del sol tal como fue transmitido por "el estudio de los ancianos". Estos lapsos siguen teniendo sentido en este nuevo contexto impuesto y se los maneja acorde a su forma de entender el movimiento del sol, armonizándolo con la construcción heredada de sus ancianos. De esta forma, la construcción idiomática ahéwa likípi que se refiere al reloj también podría asociarse perfectamente a la marcación de las horas nocturnas tomando como referencia al sol, siendo su marcador más claro la medianoche.

Junto a otros dos derivados nominales, más vinculados al uso del reloj en la muñeca de la mano yowágahe 'mi objeto femenino que está relacionada con la mano' y al hecho de que es un objeto mirado yikatená 'mi objeto femenino que miro', para nombrar al reloj utilizan la construcción idiomática ahéwa likípi, teniendo como base de sustentación "los estudios de los ancianos". Las tres maneras de referirse al reloj comparten la asignación al género femenino, género en el que es entendida la figura de sol (ahéwa) entre los grupos toba del oeste de Formosa. En suma, de toda la batería de señales con la que es posible leer el transcurso de la jornada, la construcción idiomática para nombrar al 
reloj se refiere al sol; una de las explicaciones posibles es que de todas las estrategias que utilizaban enfatizan el uso del sol puesto que es aquella forma de ritmar el tiempo que más se asemejaba al transcurso de las horas que se lee en el reloj que les es impuesto cuando empiezan a tratar con la sociedad global. De acuerdo a su perspectiva intercultural, altamente desarrollada, los Toba adoptan el reloj interpretándolo en sus propios términos, en base a "los estudios de los ancianos".

El abordaje interdisciplinario que se propone en este trabajo constituye un punto de partida para futuras investigaciones atentas a la combinación de las estrategias auditivas con las visuales en la expresión de referencia temporal en toba del oeste de Formosa y en otros pueblos indígenas del Gran Chaco.

\section{Referencias bibliográficas}

ARENAS, Pastor. Etnografía y Alimentación entre los Toba-Ñachilamole\#ek y Wichí-Lhuku'tas del Chaco Central (Argentina). Buenos Aires: Dunken, 2003.

ARENAS, Pastor; PORINI, Gustavo. Las aves en la vida de los Tobas del oeste de la provincia de Formosa (Argentina). Asunción: Tiempo de Historia, 2009.

ARNOTT, John. Los Toba-Pilagá del Chaco y sus guerras. Revista Geográfica Americana, Buenos Aires, n. 7, v. 1, p. 491-501, abr. 1934.

BAQUERO, Álvaro. Tiempo y espacio entre los Guahibo, habitantes de la Orinoquía. Scripta Ethnologica, Buenos Aires, Supplementa 9, v. 9, p. 131-142, oct. 1989.

BOOIJ, Geert. The grammar of words: an introduction to linguistic morphology. Oxford: Oxford University Press, 2007.

BOSSERT, Federico; CÓRDOBA, Lorena. El trabajo indígena en economías de enclave: una visión comparativa (Barracas caucheras e ingenios azucareros, siglos XIX y XX). In: CÓRDOBA, Lorena; BOSSERT, Federico; RICHARD, Nicolás (Org.). Capitalismo en las selvas: enclaves industriales en el Chaco y la Amazonía indígena (1850 - 1950). San Pedro de Atacama: Ediciones del Desierto, 2015. p. 111-128.

CAROL, Javier. Lengua chorote (mataguayo): estudio descriptivo. 2011. $466 \mathrm{f}$. Tesis (Doctorado en Letras) - Facultad de Filosofía y Letras, Universidad de Buenos Aires, Buenos Aires, [2011]. 
CARPIO, María Belén. Fonología y morfosintaxis de la lengua hablada por grupos tobas en el oeste de Formosa (Argentina). München: LINCOM Europa Academic Publisher, 2012. (LINCOM Studies in Native American Linguistics, 67).

"Restricted group" and "group" within the pronominal system of Western Toba (Guaicuruan, Argentina). Studies in language, Amsterdam, v. 38, n. 4, p. 982-994, 2014.

CENSABELLA, Marisa. Descripción funcional de un corpus en lengua toba (familia guaycurú, Argentina): sistema fonológico, clases sintácticas y derivación: aspectos de sincronía dinámica. 2002. 324 f. Tesis (Doctorado en Letras Modernas) - Universidad Nacional de Córdoba, Córdoba, [2002].

COMBÈS, Isabelle. ¿Quién mató a Crevaux? un asesinato en el Pilcomayo en 1882. Santa Cruz de la Sierra: El País, 2017.

COMRIE, Bernard. Ergativity. In: LEHMANN, Winfred (Org.). Syntactic typology: studies in the Phenomenology of Language. Sussex: Harvester Press, 1978. p. 329-394.

CÚNEO, Paola. Formación de palabras y clasificación nominal en el léxico etnobiológico en toba (guaycurú). München: LINCOM Europa Academic Publisher, 2013. (LINCOM Studies in Native American Linguistics, 68).

FEINSTEIN, Alejandro. Astronomía elemental. Buenos Aires: Kapelusz, 1969

FLOYD, Simeon. Modally hybrid grammar? celestial pointing for time-of-day reference in Nheengatú. Language, Whashington, v. 92, n. 1, p. 31-64, 2016.

GERZENSTEIN, Ana. Lengua chorote: estudio descriptivo-comparativo y vocabulario, 3 (1-2). Buenos Aires: Universidad de Buenos Aires, Facultad de Filosofía y Letras, Instituto de Lingüística, 1978.

Lengua chorote: variedad 2: estudio descriptivo-comparativo y vocabulario, 4. Buenos Aires: Universidad de Buenos Aires, Facultad de Filosofía y Letras, Instituto de Lingüística, 1983.

GIVÓN, Talmy. Syntax: a functional-typological introduction. Amsterdam/Philadelphia: John Benjamins Publishing Company, 2001. v. 1.

GÓMEZ, Cecilia. La luna y la feminidad entre los Tobas del oeste formoseño (Gran Chaco, Argentina). Campos, Curitiba, v. 11, n. 1. p. 47-64, 2010.

GÓMEZ, Cecilia. El hombre y la mujer: apuntes sobre la organización social toba y su relación con el ámbito celeste. Suplemento Antropológico, Asunción, v. 47, n. 2, p. 7$112,2012$. 
GORDILLO, Gastón. The bush, the plantations, and the "devils": culture and historical experience in the Argentinean Chaco. 1999. 455 f. Tesis (Doctorado en Filosfía) Graduate Department of Anthropology, University of Toronto, Toronto, [1999].

2005.

Nosotros vamos a estar aquí siempre: historias Tobas. Buenos Aires: Biblos,

HASPELMATH, Martin. Understanding Morphology. New York: Arnold Publishers \& Oxford University Press, 2002.

HUBERT, Henri. Estudio somero de la representación del tiempo en la religión y en la magia. In: HUBERT, Henri; MAUSS, Marcel (Org.). Magia y sacrificio en la historia de las religiones. Buenos Aires: Lautaro, 1946. p. 285-336.

IDOYAGA MOLINA, Anatilde. Astronomía pilagá. Scripta Ethnologica, Buenos Aires, Supplementa 9, v. 9, p. 17-28, 1989.

Modos de clasificación en la cultura Pilagá. Buenos Aires: Centro Argentino de Etnología Americana, 1995.

KLEIN, Harriet E. Manelis. Meronymy or part-whole relations in indigenous languages of Lowland South America. In: VAN DER VOORT, Hein; VAN DE KERKE, Simon (Org.). Essays on indigenous languages of Lowland South America: contributions to the 49th International Congress of Americanists in Quito 1997. The Netherlands: Universiteit Leiden, 2000. p. 83-98.

LEHMANN-NITSCHE, Roberto. La Astronomía de los Tobas (segunda parte). Revista del Museo de La Plata, Buenos Aires, v. 28, p. 181-209, 1924/5.

LÓPEZ, Alejandro Martín. La Virgen, el árbol y la serpiente: cielos e identidades en comunidades mocovíes del Chaco. 2009. 385 f. Tesis (Doctorado en Antropología) Facultad de Filosofía y Letras, Universidad de Buenos Aires, [2009].

MARTÍNEZ, Gustavo; CÚNEO, Paola. Las denominaciones vernáculas y el conocimiento toba del entorno vegetal. Revista de Dialectología y Tradiciones Populares (RDTP), v. 64, n. 2, p. 149-168, 2009.

MESSINEO, Cristina. Lengua toba (guaycurú): aspectos gramaticales y discursivos. München: LINCOM Europa Academic Publisher, 2003. (LINCOM Studies in Native American Linguistics, 48).

MÉTRAUX, Alfred. Myths of the Toba and Pilagá Indians of the Gran Chaco. Philadelphia: American Folklore Society, 1946a.

Ethnography of the Chaco. In: STEWARD, Julian (Org.). Handbook of South American Indians: the Marginal tribes. Washington: Smithsonian Institution, 1946b, p. 197-380. v. 1. 
MONTANI, Rodrigo. El mundo de las cosas entre los Wichís del Gran Chaco: un ensayo etnolingüístico. Cochabamba: Itinerarios Editorial, 2017. (Colección Scripta Autochtona, 17).

NORDENSKIÖLD, Erland. La vida de los indios. El Gran Chaco (Sudamérica). La Paz: APCOB, 2002 [1912].

PALAVECINO, Enrique. Los indios pilagá del río Pilcomayo. Anales del Museo Nacional de Historia Natural Bernardino Rivadavia, Buenos Aires, n. 37, p. 517-581, 1933.

PEDROZA LIMA, Flavia et al. Relações céu-terra entre os indígenas no Brasil: distintos céus, diferentes olhares. In: MATSUURA, Oscar (Org.). História da Astronomia no Brasil. Río de Janeiro: Museu de Astonomia e Ciências Afins/Cepe Editora, 2013. p. 86130. v. 1.

REESINK, Edwin. A preliminary note on Kanamari astronomy. Scripta Ethnologica, Buenos Aires, Supplementa 9, v. 9, p. 121-130, 1989.

STORM, Olaf. El río Pilcomayo y el Chaco boreal: viajes y exploraciones. Buenos Aires: Compañía Sudamericana de Billetes de Banco, 1892.

TOLA, Florencia. Yo no estoy solo en mi cuerpo: cuerpos-personas múltiples entre los Tobas del Chaco argentino. Buenos Aires: Biblos, 2012.

TOMASINI, Alfredo. Dapichi: un alto Dios uranio de los Toba y Pilagá. Scripta Ethnologica, Buenos Aires, v. 4, n. 1, p. 69-87, 1976.

URTON, Gary. En el cruce de rumbos de la tierra y el cielo. Cusco: Centro de Estudios Regionales Andinos Bartolomé de las Casas, 2005 [1981].

WILBERT, Johannes; SIMONEAU, Karin (Org.) Folk Literature of the Toba Indians. Volume One. Los Angeles: UCLA Latin American Center Publications. (UCLA Latin American Studies, 54), 1982.

WRIGHT, Pablo. Sueño, shamanismo y evangelio en los Qom (Tobas) del Chaco argentino. Sociedad y Religión, Buenos Aires, v. 25, n. 44, p. 30-61, 2015.

Recebido em: 03/08/2017 * Aprovado em: 17/05/2018 * Publicado em: 30/06/2018 